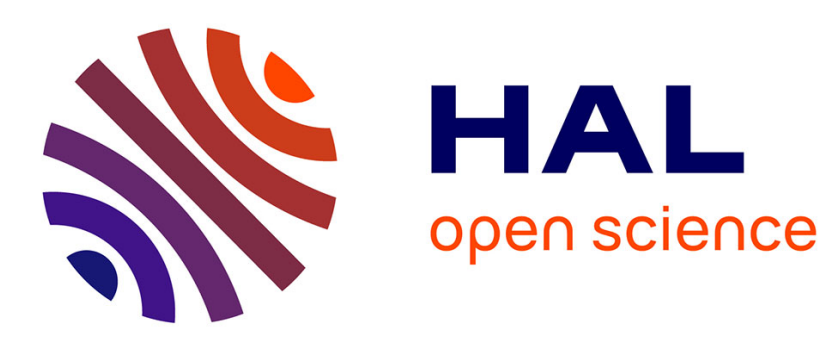

\title{
Converse Lyapunov-Krasovskii theorems for uncertain retarded differential equations
}

\author{
Ihab Haidar, Paolo Mason, Mario Sigalotti
}

\section{To cite this version:}

Ihab Haidar, Paolo Mason, Mario Sigalotti. Converse Lyapunov-Krasovskii theorems for uncertain retarded differential equations. Automatica, 2015, 62, pp.263-273. 10.1016/j.automatica.2015.09.034 . hal-00924252v3

\section{HAL Id: hal-00924252 \\ https://inria.hal.science/hal-00924252v3}

Submitted on 22 Dec 2017

HAL is a multi-disciplinary open access archive for the deposit and dissemination of scientific research documents, whether they are published or not. The documents may come from teaching and research institutions in France or abroad, or from public or private research centers.
L'archive ouverte pluridisciplinaire HAL, est destinée au dépôt et à la diffusion de documents scientifiques de niveau recherche, publiés ou non, émanant des établissements d'enseignement et de recherche français ou étrangers, des laboratoires publics ou privés. 


\title{
Converse Lyapunov-Krasovskii theorems for uncertain retarded differential equations *
}

\author{
Ihab Haidar ${ }^{\text {a }}$ Paolo Mason ${ }^{\text {a }}$ Mario Sigalotti ${ }^{b}$ \\ ${ }^{a}$ Laboratoire des Signaux et Systèmes (L2S), CNRS, CentraleSupélec, Univ Paris-Sud, Université Paris-Saclay, 91192 \\ Gif-sur-Yvette, France (e-mail: ihab.haidar@lss.supelec.fr, paolo.mason@l2s.centralesupelec.fr) \\ ${ }^{\mathrm{b}}$ Inria Saclay, team GECO \& CMAP, École Polytechnique, CNRS, Université Paris-Saclay, Palaiseau, 91128, France \\ (e-mail: mario.sigalotti@inria.fr)
}

\begin{abstract}
In this article we give a collection of converse Lyapunov-Krasovskii theorems for uncertain retarded differential equations. We show that the existence of a weakly-degenerate Lyapunov-Krasovskii functional is a necessary and sufficient condition for the global exponential stability of linear retarded functional differential equations. This is carried out using a switched system representation approach.
\end{abstract}

Key words: Time-delay system; switched systems; Lyapunov-Krasovskii functional; converse Lyapunov theorems; exponential stability.

\section{Introduction}

In the context of time-varying delay systems, many important problems concern their stability. Two principal approaches in the stability analysis are the LyapunovKrasovskii method and Lyapunov-Razumikhin method [8]. Based on these two approaches, a variety of stability criteria has been developed (see e.g. [1,3-5,16,18,20] and references therein). These criteria are often formulated as linear matrix inequalities (LMIs) which yield sufficient conditions for stability. Switched systems theory offers a complementary insight in this context. In [10], Hetel, Daafouz and Iung establish a theoretical link between the Lyapunov-Krasovskii approach and the switched system representation, in the context of discrete-time systems with time-varying delays. They prove that, looking for a delay-dependent LyapunovKrasovskii functional for the initial system, is equivalent to applying the multiple Lyapunov functions approach to the switched systems representation. This paper shares the same spirit as [10] with the significant dif-

\footnotetext{
* This work was supported by the DIGITEO Grant 2012061D SSyCoDyC and by a public grant overseen by the French National Research Agency (ANR) as part of the "Investissement d'Avenir" program, through the "iCODE Institute project" funded by the IDEX Paris-Saclay, ANR-11IDEX-0003-02.
}

ference that it considers the case of continuous-time systems with time-varying delays. An important feature of this setting is that the switched system formulation, obtained by standard functional representation, describes an evolution in an infinite-dimensional Banach space. Switched systems in Banach and Hilbert spaces have been studied for instance in $[9,21]$, where some converse Lyapunov theorems have been obtained.

In this paper we give a collection of converse LyapunovKrasovskii theorems for uncertain retarded differential equations. The term uncertain refers here to the fact that delays may vary arbitrarily in a given interval, or, more generally, that the operator describing the retarded dynamics varies arbitrarily in a given set of modes. Some of the obtained results are deduced from general necessary and sufficient conditions for stability proved in [9]. The other results exploit the specific properties of the class of systems under consideration here, which allow finer characterizations, based on ad hoc arguments. An important technical tool on which our arguments are based is an adaptation, proved in [9], of a result obtained in [22] in the framework of $C_{0}$-semigroups (see Lemma 9 below). Such a result allows to deduce exponential stability from a uniform exponential growth bound coupled with an integrability property of forward-in-time trajectories.

Several converse Lyapunov-Krasovskii theorems have been presented in the literature for systems described 
by retarded functional differential equations, in a general (possibly time-varying but not uncertain) setting $[6,11,14,19]$. In [19], in particular, the authors show that the existence of a Lyapunov-Krasovskii functional is a necessary and sufficient condition for the uniform global asymptotic stability and the global exponential stability of autonomous systems described by neutral functional differential equations in Hale's form. In [11], the author extends the well-known Lyapunov theorem given for linear ordinary differential equations to general linear time-delay systems. A method to construct a quadratic Lyapunov functional for such systems is proposed. Even though this Lyapunov functional is quadratic, it only admits a cubic lower bound. Another method to construct Lyapunov functionals for a class of linear time-delay systems is proposed in $[6,14]$. These Lyapunov functionals admit a quadratic lower bound.

More in the spirit of the results presented here are the converse Lyapunov theorems for uncertain nonlinear retarded differential equations obtained by Karafyllis and collaborators in $[12,13]$. The latter are obtained for a very general class of dynamics (which in particular are allowed to be non-autonomous). The conclusions are accordingly less detailed on the properties of the Lyapunov functionals with respect to ours, as explained in Section 4.

In this work, we consider a general linear retarded functional differential equation (RFDE)

$$
\dot{x}(t)=\Gamma(t) x_{t},
$$

where $x(t) \in \mathbb{R}^{n}, x_{t}:[-r, 0] \rightarrow \mathbb{R}^{n}$ is the standard notation for the history function defined by $x_{t}(\theta)=x(t+\theta)$, $-r \leq \theta \leq 0$. For every time $t$, the operator $L=\Gamma(t)$ associates with $x_{t}$ a vector in $\mathbb{R}^{n}$ : a typical example is $L x_{t}=A_{0} x(t)+A_{1} x(t-\tau)$ for some $n \times n$ matrices $A_{0}$ and $A_{1}$, and some delay $\tau \in[-r, 0]$. Two phase spaces are considered: $C\left([-r, 0], \mathbb{R}^{n}\right)$ and the Sobolev space $H^{1}\left([-r, 0], \mathbb{R}^{n}\right)$. The time-varying operator $\Gamma(\cdot)$ is piecewise constant or, more generally, measurable with values in a given set of operators $Q$, which is not necessarily finite nor countable. We are interested in properties that are uniform with respect to $\Gamma(\cdot)$, which plays the role of a uncertain retarded dynamics. We prove that system (1) is uniformly exponentially stable in $C\left([-r, 0], \mathbb{R}^{n}\right)$ if and only if its restriction to the $H^{1}\left([-r, 0], \mathbb{R}^{n}\right)$ is also uniformly exponentially stable (with respect to the $H^{1}$ norm). We then give a collection of converse LyapunovKrasovskii theorems. They characterize the uniform exponential stability in terms of the existence of functionals with suitable commensurability conditions and decreasing uniformly along the solutions of (1). One of the novelties of our results is that these functionals may be weakly-degenerate, i.e., they may not have a strictly positive norm-dependent lower bound, in contrast with what is known in the literature.

The paper is organized as follows. Section 2 is devoted to the problem framework. Namely, we discuss the wellposedness and the switched system representation of sys- tem (1). The statement of the main result (Theorem 2) is presented in Section 3. Section 4 is devoted to the comparison of our results with some previously known converse Lyapunov-Krasovskii theorems. An example that describes the applicability of our main result is given in Section 5 . Next, some stability properties are proved in Section 6 . The core of the proof of Theorem 2 is given in Section 7, while an application is discussed in Section 8.

\section{Problem framework}

Let $r>0$ be a real number and let $X=X\left([-r, 0], \mathbb{R}^{n}\right)$ be a linear real vector space of functions mapping $[-r, 0]$ into $\mathbb{R}^{n}$. Assume that a norm $\|\cdot\|_{X}$ is given in $X$, and assume that $\left(X,\|\cdot\|_{X}\right)$ is a Banach space. Two different choices of Banach spaces are considered: $X=\left(C\left([-r, 0], \mathbb{R}^{n}\right),\|\cdot\|_{C}\right)$ and $X=\left(H^{1}\left([-r, 0], \mathbb{R}^{n}\right),\|\cdot\|_{H^{1}}\right)$, where

$$
\begin{aligned}
\|\psi\|_{C} & =\max _{\theta \in[-r, 0]}|\psi(\theta)| \\
\|\psi\|_{H^{1}} & =\left(\int_{-r}^{0}\left(|\psi(\theta)|^{2}+\left|\frac{d}{d \theta} \psi(\theta)\right|^{2}\right) d \theta\right)^{\frac{1}{2}} .
\end{aligned}
$$

Let $Q$ be any subset of the space of linear operators from $C\left([-r, 0], \mathbb{R}^{n}\right)$ to $\mathbb{R}^{n}$. A crucial hypothesis in what follows is that $Q$ is a bounded subset of $\mathcal{L}\left(C\left([-r, 0], \mathbb{R}^{n}\right), \mathbb{R}^{n}\right)$, i.e. there exists a positive constant $m$ such that

$$
|L \psi| \leq m\|\psi\|_{C} \quad \forall \psi \in C\left([-r, 0], \mathbb{R}^{n}\right), L \in Q .
$$

Let us associate with $Q$ the linear Retarded Functional Differential Equation (RFDE)

$$
\dot{x}(t)=\Gamma(t) x_{t}, \quad t \geq t_{0},
$$

where $x:\left[t_{0}-r,+\infty\right) \rightarrow \mathbb{R}^{n}, x_{t}:[-r, 0] \rightarrow \mathbb{R}^{n}$ denotes the history function defined by

$$
x_{t}: \theta \mapsto x(t+\theta), \quad \theta \in[-r, 0], \quad t \geq t_{0},
$$

$\Gamma:\left[t_{0}, \infty\right) \rightarrow Q$ is piecewise constant (hence, with finitely many discontinuities on each bounded interval) or measurable, in the sense that $\Gamma(\cdot) \psi:\left[t_{0}, \infty\right) \rightarrow \mathbb{R}^{n}$ is measurable for any $\psi \in C\left([-r, 0], \mathbb{R}^{n}\right)$. We denote by $\mathrm{PC}\left(\left[t_{0},+\infty\right), Q\right)$ and $\mathcal{M}\left(\left[t_{0},+\infty\right), Q\right)$ (or simply $\mathrm{PC}$ and $\mathcal{M})$ the classes of, respectively, piecewise constant and measurable functions with values in $Q$.

Denote by $\varphi \in X$ the initial condition for (3) at time $t_{0}$, i.e.,

$$
x\left(t_{0}+\theta\right)=\varphi(\theta), \quad \theta \in[-r, 0] .
$$


Example 1 A particular case of system (3)-(4) is the following type of linear time-varying delayed systems

$$
\begin{aligned}
\dot{x}(t) & =\sum_{i=1}^{p} A_{\sigma(t), i} x\left(t-\tau_{i}(\sigma(t))\right), \quad t \geq t_{0}, \\
x_{t_{0}} & =\varphi \in X
\end{aligned}
$$

where $\tau_{i}(\sigma) \in[0, r]$ and $A_{\sigma, i}$ is a $n \times n$ matrix for every $\sigma$ in a given set $\Sigma$ and every $i \in\{1, \ldots, p\}$. If the set of matrices $\left\{A_{\sigma, i} \mid \sigma \in \Sigma, i \in\{1, \ldots, p\}\right\}$ is bounded, then the set $Q$ of the operators $L_{\sigma} \psi=\sum_{i=1}^{p} A_{\sigma, i} \psi\left(-\tau_{i}(\sigma)\right)$, $\sigma \in \Sigma$, is bounded in $\mathcal{L}\left(C\left([-r, 0], \mathbb{R}^{n}\right), \mathbb{R}^{n}\right)$.

Example 2 Another particular case of system (3)-(4) is the following integro-differential equation

$$
\begin{aligned}
& \dot{x}(t)=\int_{0}^{r} A_{\sigma(t), \theta} x(t-\theta) d \theta, \quad t \geq t_{0}, \\
& x_{t_{0}}=\varphi \in X,
\end{aligned}
$$

where $A_{\sigma, \theta}$ is a $n \times n$ matrix uniformly bounded with respect to $\theta \in[0, r]$ and to $\sigma$ in a given set $\Sigma$, and measurable with respect to $\theta$. In this case the set $Q$ of the operators $L_{\sigma} \psi=\int_{0}^{r} A_{\sigma, \theta} \psi(-\theta) d \theta, \sigma \in \Sigma$, is bounded in $\mathcal{L}\left(C\left([-r, 0], \mathbb{R}^{n}\right), \mathbb{R}^{n}\right)$.

\subsection{Well-posedness}

In this section we discuss the well-posedness problem for a RFDE, depending on the choice of the space $X$. Before that, we recall, following [8], that a function $x$ is said to be a solution of system (3) on $[-r,+\infty)$ with initial condition $\varphi \in X\left([-r, 0], \mathbb{R}^{n}\right)$ if $x$ is absolutely continuous on $\left[t_{0},+\infty\right), x_{t} \in X\left([-r, 0], \mathbb{R}^{n}\right)$ for every $t \geq t_{0}$ and $x(t)$ satisfies system (3) for almost every $t \geq t_{0}$.

Lemma 1 Let $X$ be the Banach space $C\left([-r, 0], \mathbb{R}^{n}\right)$ or $H^{1}\left([-r, 0], \mathbb{R}^{n}\right)$. Assume that condition (2) holds. For every $\varphi \in X$ and every $\Gamma(\cdot) \in \mathcal{M}\left(\left[t_{0},+\infty\right), Q\right)$ there exists a unique solution in $X$ of (3)-(4).

Proof. When $X=C\left([-r, 0], \mathbb{R}^{n}\right)$ and under the hypothesis given by equation (2), the existence and uniqueness of solutions for system (3)-(4) is guaranteed by [8, Theorem 1.1]. When $X=H^{1}\left([-r, 0], \mathbb{R}^{n}\right)$, one has to check that such solution, a priori defined in $C\left([-r, 0], \mathbb{R}^{n}\right)$, actually stays in $X$. This is true since both $x$ and $\dot{x}$ are locally bounded for positive times.

\subsection{Switched system representation for $\Gamma(\cdot)$ piecewise} constant

In the case where $Q$ is not bounded one can nevertheless associate with (3), when $\Gamma(\cdot) \in \operatorname{PC}\left(\left[t_{0},+\infty\right), Q\right)$, an evolution operator obtained by concatenating the flows corresponding to constant values of $\Gamma(\cdot)$. Recall that with any $L \in Q$ one can associate a $C_{0}$-semigroup $T_{L}(t): X \rightarrow X, t \geq 0$, with infinitesimal generator $\mathcal{A}_{L}$ given by

$$
\begin{aligned}
& D\left(\mathcal{A}_{L}\right)=\left\{\psi \in X: \frac{d \psi}{d \theta} \in X, \frac{d \psi}{d \theta}(0)=L \psi\right\}, \\
& \mathcal{A}_{L} \psi=\frac{d \psi}{d \theta} .
\end{aligned}
$$

By definition, $T_{L}(t)$ is the flow at time $t$ of equation (3) with $t_{0}=0$ and $\Gamma(\cdot)$ constantly equal to $L$.

The evolution operator corresponding to a piecewise constant $\Gamma(t)=\sum_{k \geq 0} \mathbb{1}_{\left[t_{k}, t_{k+1}\right)}(t) L_{k}$ with $t_{k}<t_{k+1}$ for $k \geq 0$ is given by

$$
T_{\Gamma(\cdot)}\left(t, t_{0}\right)=T_{L_{k}}\left(t-t_{k}\right) T_{L_{k-1}}\left(t_{k}-t_{k-1}\right) \cdots T_{L_{0}}\left(t_{1}-t_{0}\right)
$$

for each $t \in\left[t_{k}, t_{k+1}\right)$. This is exactly the notion of switched system

$$
\begin{aligned}
& x_{t}=T_{\Gamma(\cdot)}\left(t, t_{0}\right) x_{t_{0}}, \\
& x_{t_{0}}=\varphi \in X,
\end{aligned}
$$

considered in [9] for general Banach spaces.

\section{Remark 1 (Switched system representation)}

System (7) gives a switched system representation of the RFDE (1). This idea is inspired from [10], which deals with discrete time-varying delay systems of the form

$$
x(k+1)=A x(k)+A_{d} x(k-\tau(k)),
$$

where $0<\tau(k) \leq m$ is a positive integer, $A$ and $A_{d}$ are $n \times n$ real matrices. Following [10], one can consider the augmented finite-dimensional state vector

$$
z(k)=\left[x^{T}(k), \ldots, x^{T}(k-m)\right]^{T},
$$

which represents the history function of system (8). The delay system (8) is then rewritten as the discrete-time switched system

$$
z(k+1)=\bar{A}_{\sigma(k)} z(k),
$$

where the parameter $\sigma(k) \in\{1,2, \ldots, m\}$ switches according to the delay and $\bar{A}_{1}, \ldots, \bar{A}_{m}$ are $n m \times n m ~ m a-$ trices representing the dynamics of the history function. The switched system representation, by replacing $x(k)$ by $z(k)$, allows to get rid of the delay in the dynamics, the price being the increase of the dimension of the state space. System (7) can be seen as a continuous-time counterpart of (9). The finite-dimensional variable $x(t)$ is here replaced by the infinite-dimensional history function $x_{t}$. 
Remark 2 Similarly to what done in the case where $Q$ is not bounded, one can associate with system (5), considered in Example 1, a switched system in the $L^{2}$ setting, defined by concatenation of $C_{0}$-semigroups. For every $\sigma \in \Sigma$ and $(\varphi, z) \in L^{2}\left([-r, 0], \mathbb{R}^{n}\right) \times \mathbb{R}^{n}$ there exists a function $x(\cdot)$, absolutely continuous on $\left[t_{0},+\infty\right)$, satisfying $x\left(t_{0}\right)=z$ and (5) almost everywhere. Moreover $\left(x_{t}(\cdot), x(t)\right)=T_{\sigma}\left(t-t_{0}\right)(\varphi(\cdot), z)$ where $T_{\sigma}(\cdot)$ is a $C_{0}$-semigroup on $L^{2}\left([-r, 0], \mathbb{R}^{n}\right) \times \mathbb{R}^{n}$ (see e.g. [2]). We discuss the stability properties of this switched system in Section 6.2.

\section{Statement of the main result}

The notion of uniform exponential stability is recalled in the following definition.

Definition 1 We say that system (3) is $\mathcal{S}$-uniformly exponentially stable in $X$, with $\mathcal{S}=\mathrm{PC}\left(\left[t_{0},+\infty\right), Q\right)$ or $\mathcal{M}\left(\left[t_{0},+\infty\right), Q\right)$, if there exist two constants $\alpha \geq 1$ and $\beta>0$ such that for every initial condition $\varphi \in X$ and every $\Gamma(\cdot) \in \mathcal{S}$ the solution $x(t, \varphi)$ of (3)-(4) satisfies

$$
\left\|x_{t}\right\|_{X} \leq \alpha e^{-\beta\left(t-t_{0}\right)}\|\varphi\|_{X}, t \geq t_{0}
$$

Remark 3 Let us check that, because of its linear nature, system (3) is $\mathcal{S}$-uniformly exponentially stable if and only if it is $\mathcal{S}$-uniformly asymptotically stable. We recall that system (3) is said to be $\mathcal{S}$-uniformly asymptotically stable if, on the one hand, it is $\mathcal{S}$-uniformly stable, i.e., for every $R>0$ there exists $r>0$ such that, for every solution $x(\cdot)$ of (3)-(4) corresponding to $\Gamma(\cdot) \in \mathcal{S},\|\varphi\|_{X}<r$ implies $\left\|x_{t}\right\|_{X}<R$ for $t \geq t_{0}$, and, on the other hand, the solutions of (3)-(4) converge to zero $\mathcal{S}$-uniformly, i.e., there exists a constant $\delta>0$, independent of $t_{0}$ and $\Gamma(\cdot) \in \mathcal{S}$, such that for every $\epsilon>0$ there exists $\zeta(\epsilon)$ such that $\|\varphi\|_{X}<\delta$ implies $\left\|x_{t}\right\|_{X}<\epsilon$ for every $t \geq t_{0}+\zeta$. The equivalence is proved by taking $\bar{\zeta}=\zeta(\delta / 2)$ and noticing that, because of linearity and of the uniform convergence to zero, $\left\|x_{t}\right\| \leq 2^{-n}\|\varphi\|$ if $t \geq t_{0}+n \bar{\zeta}$, for every $n \in \mathbb{N}$. The conclusion then follows by standard arguments from the uniform stability defined above.

Remark 4 By definition of $T_{\Gamma(\cdot)}\left(t, t_{0}\right)$, we have that system (3) is $\mathrm{PC}$-uniformly exponentially stable in $X$ if and only if there exist constants $\alpha \geq 1$ and $\beta>0$ such that

$$
\left\|T_{\Gamma(\cdot)}\left(t, t_{0}\right)\right\|_{\mathcal{L}(X)} \leq \alpha e^{-\beta\left(t-t_{0}\right)}, t \geq t_{0}
$$

for every $\Gamma(\cdot) \in \mathrm{PC}\left(\left[t_{0},+\infty\right), Q\right)$. Such a uniform exponential estimate is usually taken as definition of exponential stability of the switched system (7) (see, e.g., [9]).

From now on, without loss of generality, we set $t_{0}=0$ and $T_{\Gamma(\cdot)}(t)=T_{\Gamma(\cdot)}(t, 0)$.
In order to state our main result we introduce the generalized Dini derivatives of a function $V: X \rightarrow[0, \infty)$ as follows

$$
\begin{aligned}
& \bar{D}_{L} V(\psi)=\lim _{t \rightarrow 0^{+}} \sup \frac{V\left(T_{L}(t) \psi\right)-V(\psi)}{t}, \\
& \underline{D}_{L} V(\psi)=\lim _{t \rightarrow 0^{+}} \inf \frac{V\left(T_{L}(t) \psi\right)-V(\psi)}{t},
\end{aligned}
$$

noting that possibly $\underline{D}_{L} V(\psi), \bar{D}_{L} V(\psi)=\infty$ for some $\psi \in X$ and $L \in Q$. Recall that $V(\cdot)$ is said to be directionally differentiable in the sense of Fréchet at $\psi \in X$ if there exists a positively one-homogeneous function $V^{\prime}(\psi, \cdot): X \rightarrow \mathbb{R}$ (i.e., $V^{\prime}(\psi, \lambda \xi)=\lambda V^{\prime}(\psi, \xi)$ for $\xi \in X$ and $\lambda>0)$ such that

$$
\frac{V(\psi+\xi)-V(\psi)-V^{\prime}(\psi, \xi)}{\|\xi\|_{X}} \longrightarrow 0 \quad \text { as } \xi \rightarrow 0
$$

The main result of this paper is the following theorem.

Theorem 2 Let $Q \subset \mathcal{L}\left(C\left([-r, 0], \mathbb{R}^{n}\right), \mathbb{R}^{n}\right)$ be bounded. The following statements are equivalent:

(i) System (3) is PC-uniformly exponentially stable in $C\left([-r, 0], \mathbb{R}^{n}\right)$.

(ii) System (3) is PC-uniformly exponentially stable in $H^{1}\left([-r, 0], \mathbb{R}^{n}\right)$.

(iii) System (3) is $\mathcal{M}$-uniformly exponentially stable in $C\left([-r, 0], \mathbb{R}^{n}\right)$.

(iv) System (3) is $\mathcal{M}$-uniformly exponentially stable in $H^{1}\left([-r, 0], \mathbb{R}^{n}\right)$.

(v) There exists a function $V: C\left([-r, 0], \mathbb{R}^{n}\right) \rightarrow[0, \infty)$ such that $\sqrt{V(\cdot)}$ is a norm on $C\left([-r, 0], \mathbb{R}^{n}\right)$,

$$
\underline{c}\|\psi\|_{C}^{2} \leq V(\psi) \leq \bar{c}\|\psi\|_{C}^{2}
$$

for some constants $\underline{c}, \bar{c}>0$ and

$$
\bar{D}_{L} V(\psi) \leq-\|\psi\|_{C}^{2}, \quad L \in Q, \psi \in C\left([-r, 0], \mathbb{R}^{n}\right) .
$$

(vi) There exists a directionally Fréchet differentiable function $V: H^{1}\left([-r, 0], \mathbb{R}^{n}\right) \rightarrow[0, \infty)$ such that $\sqrt{V(\cdot)}$ is a norm on $H^{1}\left([-r, 0], \mathbb{R}^{n}\right)$,

$$
\begin{aligned}
\underline{c}\|\psi\|_{H^{1}}^{2} & \leq V(\psi) \leq \bar{c}\|\psi\|_{H^{1}}^{2}, \\
\left|V^{\prime}(\psi, \xi)\right| & \leq \bar{c}\|\psi\|_{H^{1}}\|\xi\|_{H^{1}} \\
V^{\prime}\left(\psi, \xi_{1}+\xi_{2}\right) & \leq V^{\prime}\left(\psi, \xi_{1}\right)+V^{\prime}\left(\psi, \xi_{2}\right),
\end{aligned}
$$

for some constants $\underline{\underline{c}}, \bar{c}>0$ and

$$
\begin{aligned}
& \bar{D}_{L} V(\psi) \leq-\|\psi\|_{H^{1}}^{2}, \quad L \in Q, \psi \in H^{1}\left([-r, 0], \mathbb{R}^{n}\right) . \\
& \text { If } \psi \in D\left(\mathcal{A}_{L}\right) \text { then } \bar{D}_{L} V(\psi)=V^{\prime}\left(\psi, \mathcal{A}_{L} \psi\right) .
\end{aligned}
$$


(vii) There exists a continuous function

$$
V: C\left([-r, 0], \mathbb{R}^{n}\right) \rightarrow[0, \infty)
$$

such that

$$
V(\psi) \leq c\|\psi\|_{C}^{2}
$$

for some constant $c>0$ and

$$
\underline{D}_{L} V(\psi) \leq-|\psi(0)|^{2}, \quad L \in Q, \psi \in C\left([-r, 0], \mathbb{R}^{n}\right) .
$$

(viii) There exists a continuous function

$$
V: H^{1}\left([-r, 0], \mathbb{R}^{n}\right) \rightarrow[0, \infty)
$$

such that

$$
V(\psi) \leq c\|\psi\|_{H^{1}}^{2}
$$

for some constant $c>0$ and

$$
\underline{D}_{L} V(\psi) \leq-|\psi(0)|^{2}, \quad L \in Q, \psi \in H^{1}\left([-r, 0], \mathbb{R}^{n}\right) .
$$

The proof of Theorem 2 is split in different steps, as illustrated in the diagram in Figure 1.

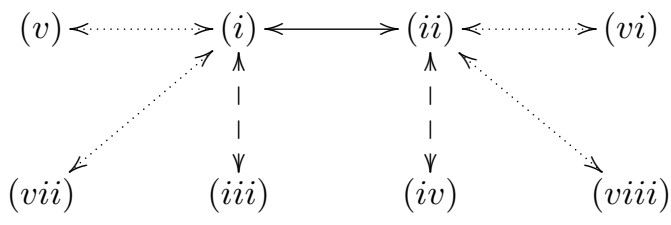

Fig. 1. Equivalences are described by different type of lines: those corresponding to continuous, dashed, and dotted lines are proved in Sections 6.3, 6.4, and 7, respectively.

Clearly, a Lyapunov function $V(\cdot)$ satisfying condition (vii) (respectively (viii)) does not necessarily satisfy the stronger conditions appearing in condition $(v)$ (respectively $(v i))$. Hence, condition (vii) (respectively (viii)) is better suited for proving the global uniform exponential stability of a RFDE, while condition $(v)$ (respectively $(v i))$ provides more information on a linear uncertain time-delay system that is known to be globally uniformly exponentially stable, by tightening the properties satisfied by $V(\cdot)$.

Remark 5 Concerning the regularity of the Lyapunov functions satisfying conditions $(v)$ and $(v i)$, recall that being a squared norm is equivalent to be positive definite, homogeneous of degree 2, continuous and convex.

\section{Discussion}

We compare here the results stated in the previous section with the Lyapunov-Krasovskii theorems given in [8] and [12]. We start by recalling a Lyapunov-Krasovskii theorem given in [8, Theorem V.2.1] for general retarded functional differential equation of the form

$$
\dot{x}=f\left(t, x_{t}\right) \text {. }
$$

Theorem 3 Suppose that $f: \mathbb{R} \times C\left([-r, 0], \mathbb{R}^{n}\right) \rightarrow \mathbb{R}^{n}$ is a continuous function. Suppose that for any bounded set $B \subset C\left([-r, 0], \mathbb{R}^{n}\right), f$ maps $\mathbb{R} \times B$ into a bounded set of $\mathbb{R}^{n}$, and $u, v, w:[0,+\infty) \rightarrow[0,+\infty)$ are continuous nondecreasing functions, $u(s)$ and $v(s)$ are positive for $s>0$, and $u(0)=v(0)=0$. If there exists a continuous function $V: \mathbb{R} \times C\left([-r, 0], \mathbb{R}^{n}\right) \rightarrow \mathbb{R}$ such that

$$
\begin{gathered}
u(|\psi(0)|) \leq V(t, \psi) \leq v\left(\|\psi\|_{C}\right) \\
\bar{D} V(t, \psi) \leq-w(|\psi(0)|)
\end{gathered}
$$

then the solution $x=0$ of equation (11) is uniformly stable. If $u(s) \rightarrow+\infty$ as $s \rightarrow+\infty$, the solutions of equation (3) are uniformly bounded. If $w(s)>0$ for $s>0$, then the solution $x=0$ is uniformly asymptotically stable.

This theorem is not given for switched systems and the uniformity property mentioned is with respect to the initial condition. The proof of Theorem 3 can be straightforwardly modified to consider linear systems of the type $(3)$, and this is reformulated by the following theorem.

Theorem 4 Assume that $Q$ is bounded in $\mathcal{L}\left(C\left([-r, 0], \mathbb{R}^{n}\right), \mathbb{R}^{n}\right)$. If there exists a continuous function $V: C\left([-r, 0], \mathbb{R}^{n}\right) \rightarrow$ $\mathbb{R}$ such that

$$
\underline{c}|\psi(0)|^{2} \leq V(\psi) \leq \bar{c}\|\psi\|_{C}^{2}
$$

for constants $\underline{\underline{c}}, \bar{c}>0$ and

$$
\bar{D}_{L} V(\psi) \leq-|\psi(0)|^{2}, \quad L \in Q, \psi \in C\left([-r, 0], \mathbb{R}^{n}\right)
$$

then system (3) is $\mathcal{M}$-uniformly exponentially stable in $C\left([-r, 0], \mathbb{R}^{n}\right)$.

Thanks to our previous results, we can give a converse version for Theorem 4. More precisely, Theorem 2 implies that if system (3) is $\mathcal{M}$-uniformly exponentially stable in $C\left([-r, 0], \mathbb{R}^{n}\right)$, then there exists a continuous function $V: C\left([-r, 0], \mathbb{R}^{n}\right) \rightarrow \mathbb{R}$ such that equations (12)-(13) hold.

Let us conclude this section by commenting on $[12,13,19]$. In $[19]$ the authors show that the existence of a Lyapunov-Krasovskii functional is a necessary and sufficient condition for the uniform global asymptotic stability and the global exponential stability of nonlinear autonomous systems described by neutral functional differential equations in Hale's form.

When restricted to linear systems of the form (3), [12, Theorem 2.10] establishes the equivalence between the uniform exponential stability in $C\left([-r, 0], \mathbb{R}^{n}\right)$ and the existence of a Lyapunov-Krasovskii functional either as in the statement (v) of Theorem 2 or with a lower bound as the one in (12). In the latter case, however, the Lyapunov-Krasovskii functional is defined on a space of the type $C\left([-r-\tau, 0], \mathbb{R}^{n}\right)$, for some $\tau \geq 0$, 
on which system (3) is lifted. Similar remarks apply to the results in [13], which extend the approach of [12] towards Lyapunov-Krasovskii characterizations of output stability.

\section{Example of weakly-degenerate Lyapunov- Krasovskii functional}

In this section we discuss through an example the absence of strictly positive norm-dependent lower bounds on $V$ in statements (vii) and (viii) of Theorem 2. The example is an evidence of the fact that the relaxed condition on $V$ might turn into an advantage while looking for Lyapunov-Krasovskii functionals.

Consider the system

$$
\begin{aligned}
& \dot{x}(t)=-x(t-r), \quad t \geq 0, \\
& x_{0}=\varphi,
\end{aligned}
$$

where $r \geq 0$ and $\varphi \in C([-r, 0], \mathbb{R})$. Let $\mathcal{A}$ be the infinitesimal generator of the $C_{0}$-semigroup associated with (14). The spectrum of $\mathcal{A}$ is discrete and is given by (see e.g. $[2])$

$$
\sigma_{p}(\mathcal{A})=\{\lambda \in \mathbb{C}: \Delta(\lambda)=0\}=\left(\lambda_{k}\right)_{k \in \mathbb{N}}
$$

where

$$
\Delta(\lambda)=\lambda+e^{-\lambda r} .
$$

An interesting property of $\sigma_{p}(\mathcal{A})$ is that

$$
\Re\left(\lambda_{k}\right) \rightarrow-\infty \text { as } k \rightarrow \infty .
$$

System (14) is exponentially stable if and only if $r<\pi / 2$ (see [8]), and in such case we can define a LyapunovKrasovskii functional $V$ as

$$
V(\psi)=\int_{0}^{+\infty}\|T(t) \psi\|_{C}^{2} d t
$$

where $T(\cdot)$ is the $C_{0}$-semigroup generated by $\mathcal{A}$. Notice that this classical choice of Lyapunov function can be generalized for exponentially stable switched systems of the type introduced in Section 2.2, taking

$$
V(\psi)=\sup _{\Gamma(\cdot) \in \mathrm{PC}} \int_{0}^{+\infty}\left\|T_{\Gamma(\cdot)} \psi\right\|_{X}^{2} d t,
$$

as shown in [9].

For each $k \in \mathbb{N}$, let $v_{k}(\theta)=e^{\lambda_{k}(r+\theta)}, \theta \in[-r, 0]$ be a (complex) eigenvector of $\mathcal{A}$ corresponding to $\lambda_{k}$.

Remark, from equation $(15)$, that $\lambda \in \sigma_{p}(\mathcal{A})$ if and only if $\bar{\lambda} \in \sigma_{p}(\mathcal{A})$. Associate with $\bar{\lambda}_{k}$ its eigenvector $\bar{v}_{k}(\theta)=$ $e^{\bar{\lambda}_{k}(r+\theta)}, \theta \in[-r, 0]$, and let $\nu_{k}=\left(v_{k}+\bar{v}_{k}\right) / 2=\Re\left(v_{k}\right) \in$ $C([-r, 0], \mathbb{R})$. Then

$$
T(t) \nu_{k}(\theta)=\frac{e^{\lambda_{k}(t+r+\theta)}+e^{\bar{\lambda}_{k}(t+r+\theta)}}{2} .
$$

Hence,

$$
\begin{aligned}
V\left(\nu_{k}\right) & =\int_{0}^{+\infty} \frac{\left\|e^{\lambda_{k}(t+r+\theta)}+e^{\bar{\lambda}_{k}(t+r+\theta)}\right\|_{C}^{2}}{4} d t \\
& \leq \int_{0}^{+\infty}\left\|e^{\Re\left(\lambda_{k}\right)(t+r+\theta)}\right\|_{C}^{2} d t \\
& =\int_{0}^{+\infty} e^{2 \Re\left(\lambda_{k}\right) t} d t=-\frac{1}{2 \Re\left(\lambda_{k}\right)} .
\end{aligned}
$$

Then $V\left(\nu_{k}\right) \rightarrow 0$ as $k \rightarrow+\infty$, as it follows from (16). On the other hand, we have $\left\|\nu_{k}\right\|_{C} \geq \nu_{k}(-r)=1$.

Then the function $V$ does not have a strictly positive $\|\cdot\|_{C}$-norm-dependent lower bound as in equation (10). Remark however that $\left|\nu_{k}(0)\right| \rightarrow 0$ as $k \rightarrow+\infty$ faster then $V\left(\nu_{k}\right)$, hence $V$ admits a $|\cdot|$-norm-dependent lower bound as in equation (12).

Another choice of Lyapunov-Krasovskii functional satisfying condition (vii) of Theorem 2 is

$$
\hat{V}(\psi)=\int_{0}^{+\infty}|(T(t) \psi)(0)|^{2} d t .
$$

In addition, by choosing $\hat{\nu}_{k}(\theta)=\Re\left(e^{\lambda_{k} \theta}\right)$, we can easily verify that $\hat{V}\left(\hat{\nu}_{k}\right) \rightarrow 0$ as $k \rightarrow+\infty$ while $\hat{\nu}_{k}(0)=1$ for every $k$. Hence $\hat{V}$ does not have a strictly positive $|\cdot|$ norm-dependent lower bound.

\section{$6 \quad$ Stability}

We begin this section by showing that, under assumption (2), the solutions of (3)-(4) are $\mathcal{M}$-uniformly exponentially bounded, i.e, there exist $M \geq 1, w>0$ such that

$$
\left\|x_{t}\right\|_{X} \leq M e^{w t}\|\varphi\|_{X}, \quad t \geq 0
$$

for every $\Gamma(\cdot) \in \mathcal{M}([0,+\infty), Q)$, in both cases where $X=C\left([-r, 0], \mathbb{R}^{n}\right)$ and $X=H^{1}\left([-r, 0], \mathbb{R}^{n}\right)$. This property, which is a necessary condition for the uniform exponential stability of (3), is not satisfied when $X=$ $L^{2}\left([-r, 0], \mathbb{R}^{n}\right)$, as we show by means of a counterexample.

\subsection{Uniform exponential boundedness}

The $\mathcal{M}$-uniform exponential boundedness of the solutions of (3) is given by the following lemma. 
Lemma 5 Let $Q \subset \mathcal{L}\left(C\left([-r, 0], \mathbb{R}^{n}\right), \mathbb{R}^{n}\right)$ be bounded. If $X=C\left([-r, 0], \mathbb{R}^{n}\right)$ or $H^{1}\left([-r, 0], \mathbb{R}^{n}\right)$ then the solutions of (3) are $\mathcal{M}$-uniformly exponentially bounded.

Proof. The proof is provided separately for the two cases.

Let $X=C\left([-r, 0], \mathbb{R}^{n}\right)$ and $\varphi \in X$. By integrating system (3)-(4) and using equation (2), one has for every $t \geq 0$ and every $\mu \in[-r, 0]$

$$
\begin{aligned}
|x(t+\mu)| & \leq \max \left\{\|\varphi\|_{C},|\varphi(0)|+\int_{0}^{\max \{0, t+\mu\}} m\left\|x_{s}\right\|_{C} d s\right\} \\
& \leq\|\varphi\|_{C}+m \int_{0}^{\max \{0, t+\mu\}}\left\|x_{s}\right\|_{C} d s
\end{aligned}
$$

that is

$$
\left\|x_{t}\right\|_{C} \leq\|\varphi\|_{C}+m \int_{0}^{t}\left\|x_{s}\right\|_{C} d s .
$$

Thanks to Gronwall's Lemma, we have

$$
\left\|x_{t}\right\|_{C} \leq\|\varphi\|_{C} e^{m t}
$$

which implies that the solutions of (3) are $\mathcal{M}$-uniformly exponentially bounded in $C\left([-r, 0], \mathbb{R}^{n}\right)$.

Let now $X=H^{1}\left([-r, 0], \mathbb{R}^{n}\right)$ and $\varphi \in X$. For each $t \geq 0$, we have

$$
\left\|x_{t}\right\|_{L^{2}}^{2}=\int_{-r}^{0}\left|x_{t}(s)\right|^{2} d s \leq \int_{-r}^{0}\left\|x_{t}\right\|_{C}^{2} d s \leq r\left\|x_{t}\right\|_{C}^{2} .
$$

Combining equations (19) and (20) and using the Poincaré inequality

$$
\|\varphi\|_{C} \leq \lambda_{r}\|\varphi\|_{H^{1}}
$$

where $\lambda_{r}$ only depends on $r$, gives

$$
\left\|x_{t}\right\|_{L^{2}}^{2} \leq r \lambda_{r}^{2} e^{2 m t}\|\varphi\|_{H^{1}}^{2}
$$

On the other hand, from (2), (3) and (19) we have the following inequalities

$$
\begin{aligned}
\left\|\dot{x}_{t}\right\|_{L^{2}}^{2} & =\int_{-r}^{0}\left|\dot{x}_{t}(s)\right|^{2} d s=\int_{t-r}^{t}|\dot{x}(s)|^{2} d s \\
& \leq\|\dot{\varphi}\|_{L^{2}}^{2}+\int_{0}^{t}|\dot{x}(s)|^{2} d s \\
& \leq\|\varphi\|_{H^{1}}^{2}+m^{2} \int_{0}^{t}\left\|x_{s}\right\|_{C}^{2} d s \\
& \leq\|\varphi\|_{H^{1}}^{2}+m^{2}\|\varphi\|_{C}^{2} \int_{0}^{t} e^{2 m s} d s \\
& \leq\left(1+\frac{m \lambda_{r}^{2}}{2}\left(e^{2 m t}-1\right)\right)\|\varphi\|_{H^{1}}^{2} \\
& \leq\left(1+m \lambda_{r}^{2}\right) e^{2 m t}\|\varphi\|_{H^{1}}^{2} .
\end{aligned}
$$

Combining equations (22) and (23) gives

$$
\left\|x_{t}\right\|_{H^{1}} \leq C e^{m t}\|\varphi\|_{H^{1}}
$$

with

which ends the proof.

$$
C=\sqrt{1+m \lambda_{r}^{2}+r \lambda_{r}^{2}},
$$

6.2 Uniform exponential boundedness: case $X=$ $L^{2}\left([-r, 0], \mathbb{R}^{n}\right) \times \mathbb{R}^{n}$

The uniform exponential boundedness of the solutions of (3) is not, in general, an acquired property. In fact, even if this property holds when $X=C\left([-r, 0], \mathbb{R}^{n}\right)$ or $X=H^{1}\left([-r, 0], \mathbb{R}^{n}\right)$, this is no longer true in the case $X=L^{2}\left([-r, 0], \mathbb{R}^{n}\right) \times \mathbb{R}^{n}$. This point is clarified by means of the following counterexample. Consider the switched delay system in $L^{2}([-1,0], \mathbb{R}) \times \mathbb{R}$ given by

$$
\begin{aligned}
& \dot{x}(t)=-x(t-\sigma(t)), \quad t \geq 0, \\
& x_{0}=\varphi \\
& x(0)=\varphi_{0} .
\end{aligned}
$$

According to [2, Theorem 2.4.1], for every $\left(\varphi, \varphi_{0}\right) \in$ $L^{2}([-1,0], \mathbb{R}) \times \mathbb{R}$ and every $\sigma \in \operatorname{PC}([0,+\infty),[0,1])$, there exists a unique absolutely continuous solution of (24). Notice that, for every fixed $\sigma \in[0,1]$, the operator $L_{\sigma}: \varphi \mapsto-\varphi(-\sigma)$ is unbounded when considered on $L^{2}\left([-1,0], \mathbb{R}^{n}\right)$ with values in $\mathbb{R}^{n}$.

Let, for every $p \geq 1$,

$$
\sigma_{p}(t)=\left\{\begin{array}{lll}
\sum_{k=1}^{p} a_{k} 1_{\mathcal{A}_{k}}(t)+\frac{1}{2} & \text { for } & 0 \leq t<1 / 2 \\
0 & \text { for } & t \geq 1 / 2
\end{array}\right.
$$

with $\mathcal{A}_{k}=\left[a_{k}, a_{k+1}\right), a_{k}=\frac{k-1}{2 p}, k=1, \cdots, p+1$, and let

$$
\varphi_{0}=0, \quad \varphi(s)=\left|s+\frac{1}{2}\right|^{\alpha}, \quad-1 \leq s<0,
$$

where $\alpha$ is a real number such that $\alpha \in(-1 / 2,0)$. The solutions of (24) corresponding to $\sigma=\sigma_{p}, p \in \mathbb{N}$, are not uniformly exponentially bounded with respect to the choice of $p$. In fact, one can easily verify that, for $t \leq 1 / 2$,

$$
x(t)=-\int_{0}^{t} \varphi\left(s-\sigma_{p}(s)\right) d s .
$$

Since

$$
-\frac{1}{2} \leq s-\sigma_{p}(s)<\frac{1}{2 p}-\frac{1}{2} \quad \forall s \in \mathcal{A}_{k},
$$


we obtain the inequality

$|x(t)|=\int_{0}^{t} \varphi\left(s-\sigma_{p}(s)\right) d s>\int_{0}^{t}\left(\frac{1}{2 p}\right)^{\alpha} d s=\frac{t}{(2 p)^{\alpha}}$.

As a consequence, for $t \leq 1 / 2$, we have that

$$
\left\|x_{t}\right\|_{L^{2}} \geq \frac{t}{(2 p)^{\alpha}}
$$

Letting $p$ tend to $+\infty$, one clearly cannot find two constants $M \geq 1$ and $w>0$ such that inequality (18) holds for every $0<t \leq 1 / 2$.

Since the solutions of system (24) are not uniformly exponentially bounded, then the system cannot be uniformly exponentially stable in $L^{2}([-1,0], \mathbb{R}) \times \mathbb{R}$. Notice, however, that every solution of system (24) converges to zero in $L^{2}([-1,0], \mathbb{R}) \times \mathbb{R}$ (not uniformly with respect to $\sigma(\cdot))$. Indeed, by considering as initial condition at time $t_{0}=1$ the continuous function $x_{1}$, we may interpret equation (24) as a switched delay system in $C([-1,0], \mathbb{R})$. For this system we can apply [8, Theorem V.5.2], which ensures the uniform asymptotic stability in $C([-1,0], \mathbb{R})$. Since uniform convergence implies convergence in $L^{2}$, we conclude that $\left(x_{t}, x(t)\right) \rightarrow 0$ as $t \rightarrow+\infty$ in $L^{2}([-1,0], \mathbb{R}) \times \mathbb{R}$.

\subsection{Equivalence between exponential stability in} $C\left([-r, 0], \mathbb{R}^{n}\right)$ and in $H^{1}\left([-r, 0], \mathbb{R}^{n}\right)$

The equivalence between the exponential stability in $C\left([-r, 0], \mathbb{R}^{n}\right)$ and in $H^{1}\left([-r, 0], \mathbb{R}^{n}\right)$ of system $(3)$ is given by the following proposition.

Proposition 6 Let $Q \subset \mathcal{L}\left(C\left([-r, 0], \mathbb{R}^{n}\right), \mathbb{R}^{n}\right)$ be bounded and $\mathcal{S}=\operatorname{PC}([0,+\infty), Q)$ or $\mathcal{S}=\mathcal{M}([0,+\infty), Q)$. The following two statements are equivalent:

(a) System (3) is $\mathcal{S}$-uniformly exponentially stable in $C\left([-r, 0], \mathbb{R}^{n}\right)$

(b) System (3) is $\mathcal{S}$-uniformly exponentially stable in $H^{1}\left([-r, 0], \mathbb{R}^{n}\right)$

Proof. Firstly, remark that we have the following norm comparison

$$
\left\|x_{t}\right\|_{H^{1}} \leq \gamma\left\|x_{t}\right\|_{C[-2 r, 0]}, \quad t \geq r
$$

with $\gamma=\sqrt{r\left(1+m^{2}\right)}$, as it follows from the two estimates

$$
\left\|x_{t}\right\|_{L^{2}}^{2}=\int_{-r}^{0}\left|x_{t}(s)\right|^{2} d s \leq r\left\|x_{t}\right\|_{C[-r, 0]}^{2}
$$

and

$$
\left\|\dot{x}_{t}\right\|_{L^{2}}^{2}=\int_{t-r}^{t}\left|L_{\sigma(s)} x_{s}\right|^{2} d s \leq r m^{2}\left\|x_{t}\right\|_{C[-2 r, 0]}^{2} .
$$

Now, assume that system $(3)$ is $\mathcal{S}$-uniformly exponentially stable in $C\left([-r, 0], \mathbb{R}^{n}\right)$. Then, there exist two constants $\alpha \geq 1$ and $\beta>0$ such that, for every $\varphi \in$ $C\left([-r, 0], \mathbb{R}^{n}\right)$ and every $\Gamma(\cdot) \in \mathcal{S}$, the solution $x(\cdot)$ to (3)-(4) satisfies

$$
\left\|x_{t}\right\|_{C[-r, 0]} \leq \alpha e^{-\beta t}\|\varphi\|_{C[-r, 0]} .
$$

Then, for $t \geq r$, we have

$$
\left\|x_{t-r}\right\|_{C[-r, 0]} \leq \alpha e^{-\beta(t-r)}\|\varphi\|_{C[-r, 0]},
$$

which can be rewritten as

$$
\left\|x_{t}\right\|_{C[-2 r,-r]} \leq \alpha e^{\beta r} e^{-\beta t}\|\varphi\|_{C[-r, 0]}
$$

Combining (26) and (27) gives

$$
\left\|x_{t}\right\|_{C[-2 r, 0]} \leq \alpha e^{\beta r} e^{-\beta t}\|\varphi\|_{C[-r, 0]} .
$$

The $\mathcal{S}$-uniform exponential stability in $H^{1}\left([-r, 0], \mathbb{R}^{n}\right)$ derives from equations (25) and (28) together with (21). Conversely, assume that system $(3)$ is $\mathcal{S}$-uniformly exponentially stable in $H^{1}\left([-r, 0], \mathbb{R}^{n}\right)$. Then, there exist two constants $\alpha \geq 1$ and $\beta>0$ such that, for every $\eta \in H^{1}\left([-r, 0], \mathbb{R}^{n}\right)$ and every $\Gamma(\cdot) \in \mathcal{S}$, the solution $\hat{x}(\cdot)$ of $(3)$ with initial condition $\hat{x}_{t_{0}}=\eta$ satisfies

$$
\left\|\hat{x}_{t}\right\|_{H^{1}} \leq \alpha e^{-\beta\left(t-t_{0}\right)}\|\eta\|_{H^{1}}, t \geq t_{0} .
$$

Let $x(\cdot)$ be a solution of (3) with initial condition $x_{0}=\varphi \in C\left([-r, 0], \mathbb{R}^{n}\right)$. From (25), one has that $x_{r} \in H^{1}\left([-r, 0], \mathbb{R}^{n}\right)$. For $t \geq r$ it follows from $(29)$ that

$$
\left\|x_{t}\right\|_{H^{1}} \leq \alpha e^{-\beta(t-r)}\left\|x_{r}\right\|_{H^{1}}
$$

which implies, together with (25), that

$$
\left\|x_{t}\right\|_{H^{1}} \leq \alpha \gamma e^{-\beta(t-r)}\left\|x_{r}\right\|_{C[-2 r, 0]} .
$$

Furthermore, from (19), we have that

$$
\left\|x_{r}\right\|_{C[-2 r, 0]} \leq e^{m r}\|\varphi\|_{C[-r, 0]}
$$

Combining (31) and (32) together with (21) gives the inequalities

$$
\begin{aligned}
\left\|x_{t}\right\|_{C[-r, 0]} & \leq \lambda_{r}\left\|x_{t}\right\|_{H^{1}} \\
& \leq \alpha \gamma \lambda_{r} e^{(m+\beta) r} e^{-\beta t}\|\varphi\|_{C[-r, 0]},
\end{aligned}
$$

which end the proof. 
6.4 Equivalence between PC-uniform exponential stability and $\mathcal{M}$-uniform exponential stability

The goal of this section is to prove the following theorem.

Theorem 7 Let $Q \subset \mathcal{L}\left(C\left([-r, 0], \mathbb{R}^{n}\right), \mathbb{R}^{n}\right)$ be bounded. Let $X$ be equal to $C\left([-r, 0], \mathbb{R}^{n}\right)$ or $H^{1}\left([-r, 0], \mathbb{R}^{n}\right)$. System (3) is $\mathcal{M}$-uniformly exponentially stable if and only if it is PC-uniformly exponentially stable.

Proof. According to Proposition 6, it is enough to prove the theorem in the case $X=C\left([-r, 0], \mathbb{R}^{n}\right)$.

Let $x(\cdot)$ be a solution of (3)-(4) associated with $\Gamma(\cdot) \in \mathcal{M}([0,+\infty), Q)$ and let $T>0$. For $k \geq 1$, consider the function $\Gamma(\cdot) x_{j \frac{T}{k}} \in L^{\infty}\left(I_{j}, \mathbb{R}^{n}\right)$ with $I_{j}=\left[(j-1) \frac{T}{k}, j \frac{T}{k}\right), 1 \leq j \leq k$. Remark that $\frac{k}{T} \int_{I_{j}} \Gamma(s) x_{j \frac{T}{k}} d s$ is in the convex hull of

$$
E=\left\{L x_{j \frac{T}{k}}: L \in Q\right\} \subset \mathbb{R}^{n} .
$$

Carathéodory's theorem implies that $\frac{k}{T} \int_{I_{j}} \Gamma(s) x_{j \frac{T}{k}} d s$ is a convex combination of $n+1$ points of $E$, i.e.

$$
\frac{k}{T} \int_{I_{j}} \Gamma(s) x_{j \frac{T}{k}} d s=\sum_{i=1}^{n+1} \alpha_{i j} L_{i j} x_{j \frac{T}{k}}
$$

where $L_{i j} \in Q, \alpha_{i j}$ is nonnegative and $\sum_{i=1}^{n+1} \alpha_{i j}=1$. We use the coefficients of this convex combination to define the following function

$$
\Gamma_{k}(t)=L_{i j}, \quad t \in I_{i j}
$$

where the intervals $I_{i j}$ are such that $\bigcup_{i=1}^{n+1} I_{i j}=I_{j}$ and $\left|I_{i j}\right|=\alpha_{i j} \frac{T}{k}$. We clearly have that $\Gamma_{k}(\cdot)$ is in the space $\mathrm{PC}([0, T], Q)$ of piecewise constant functions defined in $[0, T]$ and taking values in $Q$.

Associate with $\Gamma_{n}(\cdot)$ the linear retarded functional differential equation

$$
\begin{aligned}
& \dot{x}_{k}(t)=\Gamma_{k}(t) x_{k, t}, \\
& x_{k, 0}=\varphi
\end{aligned}
$$

where $x_{k, t}:[-r, 0] \rightarrow \mathbb{R}^{n}$ is defined by $x_{k, t}(\theta)=x_{k}(t+$ $\theta),-r \leq \theta \leq 0$. Then

$$
\left\|x_{T}\right\|_{C}=\lim _{k \rightarrow+\infty}\left\|x_{k, T}\right\|_{C}
$$

as it is proved in Lemma 15 in the Appendix.
Suppose that system (3) is PC-uniformly exponentially stable. Then, there exist constants $a \geq 1$ and $b>0$ such that for every initial condition $\varphi \in C\left([-r, 0], \mathbb{R}^{n}\right)$ we have

$$
\left\|x_{k, T}\right\|_{C} \leq a e^{-b T}\|\varphi\|_{C}
$$

Combining equations (34) and (35) gives

$$
\left\|x_{T}\right\|_{C} \leq a e^{-b T}\|\varphi\|_{C}
$$

Remark that such an inequality is true for all $T>0$, concluding the proof.

\section{Sufficient conditions for stability: weakly- degenerate Lyapunov-Krasovskii functionals}

Based on the equivalence result proved in the previous section, we consider from now on only piecewise constant signals $\Gamma(\cdot)$. This allows us to exploit the switched system representation presented in Section 2.2 and apply general converse Lyapunov results obtained in the Banach setting in [9].

The first result of this section is a straightforward application of [9, Theorem 3], which gives a necessary and sufficient condition for the uniform exponential stability of switched systems of the type (7).

Proposition 8 Let $Q \quad \subset \quad \mathcal{L}\left(C\left([-r, 0], \mathbb{R}^{n}\right), \mathbb{R}^{n}\right)$ be bounded. System (3) is PC-uniformly exponentially stable in $X$ if and only if there exists a function $V: X \rightarrow[0, \infty)$ such that $\sqrt{V(\cdot)}$ is a norm on $X$,

$$
V(\psi) \leq c\|\psi\|_{X}^{2}
$$

for some constant $c>0$ and

$$
\underline{D}_{L} V(\psi) \leq-\|\psi\|_{X}^{2}, \quad L \in Q, \psi \in X \text {. }
$$

Proof. Due to the PC-uniform exponential boundedness in $X$ of the solutions of (3), obtained in Lemma 5, we can apply [9, Theorem 3], which leads to the desired equivalence.

The crucial step for the second equivalence is given by the following lemma proved in [9], which extends a result obtained in [22] in the framework of $C_{0}$-semigroups to switched systems of the form (7).

Lemma 9 Assume that

(a) there exist $M \geq 1$ and $w>0$ such that

$$
\left\|T_{\Gamma(\cdot)}(t)\right\|_{\mathcal{L}(X)} \leq M e^{w t}, \quad t \geq 0,
$$

for every $\Gamma(\cdot) \in \mathrm{PC}$; 
(b) there exist $c \geq 0$ and $p \in[1,+\infty)$ such that

$$
\int_{0}^{+\infty}\left\|T_{\Gamma(\cdot)}(t) \psi\right\|_{X}^{p} \leq c\|\psi\|_{X}^{p}
$$

for every $\psi \in X$ and every $\Gamma(\cdot) \in \mathrm{PC}$.

Then there exist $K \geq 1$ and $\mu>0$ such that

$$
\left\|T_{\Gamma(\cdot)}(t)\right\|_{\mathcal{L}(X)} \leq K e^{-\mu t}, \quad t \geq 0
$$

for every $\Gamma(\cdot) \in \mathrm{PC}$.

We are now ready to prove the following equivalence result, which introduces Lyapunov-Krasovskii functionals satisfying weakened conditions adapted to the RFDE setting.

Theorem 10 Let $Q \quad \subset \quad \mathcal{L}\left(C\left([-r, 0], \mathbb{R}^{n}\right), \mathbb{R}^{n}\right)$ be bounded. Then system (3) is PC-uniformly exponentially stable in $H^{1}\left([-r, 0], \mathbb{R}^{n}\right)$ if and only if there exists a continuous function $V: H^{1}\left([-r, 0], \mathbb{R}^{n}\right) \rightarrow[0,+\infty)$ such that

$$
V(\psi) \leq c\|\psi\|_{H^{1}}^{2}
$$

for some constant $c>0$ and

$$
\underline{D}_{L} V(\psi) \leq-|\psi(0)|^{2}, L \in Q, \psi \in H^{1}\left([-r, 0], \mathbb{R}^{n}\right) .
$$

Proof. Suppose that there exist a continuous function $V: H^{1}\left([-r, 0], \mathbb{R}^{n}\right) \rightarrow[0,+\infty)$ such that conditions (36)-(37) hold. From Lemma 5, we know that the solutions of system (3)-(4) are PC-uniformly exponentially bounded. Then, thanks to Lemma 9 , it suffices to prove that there exists a constant $c_{0}>0$ such that

$$
\int_{0}^{+\infty}\left\|x_{t}\right\|_{X}^{2} d s \leq c_{0}\|\varphi\|_{X}^{2}, \varphi \in X, \Gamma(\cdot) \in \mathrm{PC}
$$

to conclude the global exponential stability of system (3). For all $\Gamma(\cdot) \in \mathrm{PC}, \varphi \in H^{1}\left([-r, 0], \mathbb{R}^{n}\right)$ and $t \geq 0$, we have

$$
V\left(x_{t}\right)-V(\varphi) \leq-\int_{0}^{t}\left|x_{s}(0)\right|^{2} d s
$$

as it follows from (37) and the properties of the Dini derivative (see, e.g., [7]). Using the fact that $V$ is nonnegatively valued, one deduces from (36) and (39) the following inequality

$$
\int_{0}^{+\infty}\left|x_{s}(0)\right|^{2} d s \leq c\|\varphi\|_{H^{1}}^{2}
$$

In the sequel, we deduce from (40) that equation (38) holds. Firstly, remark that

$$
\begin{aligned}
\int_{0}^{t}\left\|x_{s}\right\|_{L^{2}}^{2} d s & =\int_{0}^{t} d s \int_{-r}^{0}\left|x_{s}(\theta)\right|^{2} d \theta \\
& =\int_{0}^{t} d s \int_{-r}^{0}\left|x_{s+\theta}(0)\right|^{2} d \theta \\
& =\int_{-r}^{0} d \theta \int_{0}^{t}\left|x_{s+\theta}(0)\right|^{2} d s \\
& \leq \int_{-r}^{0} d \theta \int_{-r}^{t}\left|x_{s}(0)\right|^{2} d s \\
& =r \int_{-r}^{t}\left|x_{s}(0)\right|^{2} d s .
\end{aligned}
$$

Furthermore, we have

$$
\begin{aligned}
\int_{0}^{t}\left\|\dot{x}_{s}\right\|_{L^{2}}^{2} d s & \leq r \int_{-r}^{t}\left|\dot{x}_{s}(0)\right|^{2} d s \\
& =r \int_{-r}^{t}|\dot{x}(s)|^{2} d s \\
& =r \int_{-r}^{0}|\dot{\varphi}(s)|^{2} d s+r \int_{0}^{t}|\dot{x}(s)|^{2} d s \\
& \leq r\|\varphi\|_{H^{1}}^{2}+r \int_{0}^{t}|\dot{x}(s)|^{2} d s .
\end{aligned}
$$

Let $s \geq 0$. There exists $\tau \in[-r, 0]$ such that $\left\|x_{s}\right\|_{C}=$ $|x(s+\bar{\tau})|$. Let $N \geq 1$ be a natural number. There exists $1 \leq j(s) \leq N$ such that

$$
0 \leq \tau+j(s) \frac{r}{N} \leq \frac{r}{N}
$$

We have that

$$
\begin{aligned}
|x(s+\tau)| & \leq\left|x\left(s-j(s) \frac{r}{N}\right)\right|+\int_{s-j(s) \frac{r}{N}}^{s+\tau}|\dot{x}(\theta)| d \theta \\
& \leq\left|x\left(s-j(s) \frac{r}{N}\right)\right|+\sqrt{\frac{r}{N}}\|\dot{x}\|_{L^{2}\left[s-j(s) \frac{r}{N}, s+\tau\right]} \\
& \leq\left|x\left(s-j(s) \frac{r}{N}\right)\right|+\sqrt{\frac{r}{N}}\left\|\dot{x}_{s}\right\|_{L^{2}[-r, 0]} \\
& \leq\left|x\left(s-j(s) \frac{r}{N}\right)\right|+\sqrt{\frac{r}{N}}\left\|x_{s}\right\|_{H^{1}} .
\end{aligned}
$$

Therefore, from equation (2), we have

$$
\begin{aligned}
|\dot{x}(s)| & \leq m\left|x\left(s-j(s) \frac{r}{N}\right)\right|+m \sqrt{\frac{r}{N}}\left\|x_{s}\right\|_{H^{1}} \\
& \leq m \sum_{j=1}^{N}\left|x\left(s-j \frac{r}{N}\right)\right|+m \sqrt{\frac{r}{N}}\left\|x_{s}\right\|_{H^{1}},
\end{aligned}
$$


from which we conclude that

$$
|\dot{x}(s)|^{2} \leq 2 N m^{2} \sum_{j=1}^{N}\left|x\left(s-j \frac{r}{N}\right)\right|^{2}+2 m^{2} \frac{r}{N}\left\|x_{s}\right\|_{H^{1}}^{2} .
$$

By integrating the previous equation over $[0, t]$, we obtain

$$
\begin{aligned}
\int_{0}^{t}|\dot{x}(s)|^{2} d s \leq & 2 N m^{2} \sum_{j=1}^{N} \int_{0}^{t}\left|x\left(s-j \frac{r}{N}\right)\right|^{2} d s \\
& +2 m^{2} \frac{r}{N} \int_{0}^{t}\left\|x_{s}\right\|_{H^{1}}^{2} d s \\
\leq & 2 N^{2} m^{2} \int_{-r}^{t}|x(s)|^{2} d s \\
& +2 m^{2} \frac{r}{N} \int_{0}^{t}\left\|x_{s}\right\|_{H^{1}}^{2} d s
\end{aligned}
$$

which implies that

$$
\begin{aligned}
\int_{0}^{t}|\dot{x}(s)|^{2} d s \leq & 2 N^{2} m^{2}\|\varphi\|_{H^{1}}^{2}+2 N^{2} m^{2} \int_{0}^{t}|x(s)|^{2} d s \\
& +2 m^{2} \frac{r}{N} \int_{0}^{t}\left\|x_{s}\right\|_{H^{1}}^{2} d s
\end{aligned}
$$

Equations (42) and (43) together imply that

$$
\begin{aligned}
\int_{0}^{t}\|\dot{x}(s)\|_{L^{2}}^{2} d s \leq & r\left(1+2 N^{2} m^{2}\right)\|\varphi\|_{H^{1}}^{2} \\
& +2 r N^{2} m^{2} \int_{0}^{t}|x(s)|^{2} d s \\
& +2 m^{2} \frac{r^{2}}{N} \int_{0}^{t}\left\|x_{s}\right\|_{H^{1}}^{2} d s .
\end{aligned}
$$

Furthermore, equations (41) and (44) together yield

$$
\begin{aligned}
\int_{0}^{t}\left\|x_{s}\right\|_{H^{1}}^{2} d s \leq & 2 r\left(1+N^{2} m^{2}\right)\|\varphi\|_{H^{1}}^{2} \\
& +r\left(1+2 N^{2} m^{2}\right) \int_{0}^{t}|x(s)|^{2} d s \\
& +2 m^{2} \frac{r^{2}}{N} \int_{0}^{t}\left\|x_{s}\right\|_{H^{1}}^{2} d s .
\end{aligned}
$$

For sufficiently large $N$, the quantity $1-2 m^{2} \frac{r^{2}}{N}$ is positive and we have

$$
\int_{0}^{t}\left\|x_{s}\right\|_{H^{1}}^{2} d s \leq c_{1} \int_{0}^{t}|x(s)|^{2} d s+c_{2}\|\varphi\|_{H^{1}}^{2} d s,
$$

with

$$
c_{1}=\frac{r\left(1+2 N^{2} m^{2}\right)}{1-2 m^{2} \frac{r^{2}}{N}} \quad \text { and } \quad c_{2}=\frac{2 r\left(1+N^{2} m^{2}\right)}{1-2 m^{2} \frac{r^{2}}{N}} .
$$

Equations (40) and (45) together imply that

$$
\int_{0}^{t}\left\|x_{s}\right\|_{H^{1}}^{2} d s \leq c_{0}\|\varphi\|_{H^{1}}^{2}
$$

with $c_{0}=c c_{1}+c_{2}$. Letting $t$ tend to $+\infty$, we deduce that

$$
\int_{0}^{+\infty}\left\|x_{t}\right\|_{H^{1}}^{2} d s \leq c_{0}\|\varphi\|_{H^{1}}^{2}
$$

which concludes, thanks to Lemma 9, the proof of the PC-uniform exponential stability of system (3) in the case when $X=H^{1}\left([-r, 0], \mathbb{R}^{n}\right)$.

Conversely, suppose that system (3) is PC-uniformly exponentially stable in $H^{1}\left([-r, 0], \mathbb{R}^{n}\right)$. Then, from Proposition 8 , there exists a function $V: H^{1}\left([-r, 0], \mathbb{R}^{n}\right) \rightarrow$ $[0, \infty)$ such that $\sqrt{V(\cdot)}$ is a norm on $H^{1}\left([-r, 0], \mathbb{R}^{n}\right)$,

$$
V(\psi) \leq c\|\psi\|_{H^{1}}^{2}
$$

for some constant $c>0$ and

$$
\underline{D}_{L} V(\psi) \leq-\|\psi\|_{H^{1}}^{2}, L \in Q, \psi \in H^{1}\left([-r, 0], \mathbb{R}^{n}\right) .
$$

Thanks to the Poincaré inequality (21) and the fact that $|\psi(0)| \leq\|\psi\|_{C}$, equation (47) yields

$$
\underline{D}_{L} V(\psi) \leq-\lambda_{r}|\psi(0)|^{2}, \quad L \in Q, \psi \in H^{1}\left([-r, 0], \mathbb{R}^{n}\right),
$$

which ends the proof.

The extension of this equivalence result to the case when $X=C\left([-r, 0], \mathbb{R}^{n}\right)$ is given by the following corollary.

Corollary 11 Let $Q \quad \subset \quad \mathcal{L}\left(C\left([-r, 0], \mathbb{R}^{n}\right), \mathbb{R}^{n}\right) \quad$ be bounded. System (3) is $\mathrm{PC}-$ uniformly exponentially stable in $C\left([-r, 0], \mathbb{R}^{n}\right)$ if and only if there exists a continuous function $V: C\left([-r, 0], \mathbb{R}^{n}\right) \rightarrow[0,+\infty)$ such that

$$
V(\psi) \leq c\|\psi\|_{C}^{2}
$$

for some constant $c>0$ and

$$
\underline{D}_{L} V(\psi) \leq-|\psi(0)|^{2}, \quad L \in Q, \psi \in C\left([-r, 0], \mathbb{R}^{n}\right) .
$$

Proof. Suppose that system (3) is PC-uniformly exponentially stable in $C\left([-r, 0], \mathbb{R}^{n}\right)$. Then, following the same reasoning as in Theorem 10, we know that there exists a function $V: C\left([-r, 0], \mathbb{R}^{n}\right) \rightarrow[0, \infty)$ that satisfies conditions (48)-(49).

Conversely, suppose that conditions (48)-(49) are satisfied. Using the Poincaré inequality (21), we deduce from (48) that

$$
V(\psi) \leq c_{1}\|\psi\|_{H^{1}}^{2}
$$


for some constant $c_{1}>0$ and every $\psi \in H^{1}\left([-r, 0], \mathbb{R}^{n}\right)$. Equations (49) and (50) together with Theorem 10 imply that system (3) is PC-uniformly exponentially stable in $H^{1}\left([-r, 0], \mathbb{R}^{n}\right)$. Finally, we deduce, from Proposition 6 , that system (3) is PC-uniformly exponentially stable in $C\left([-r, 0], \mathbb{R}^{n}\right)$, concluding the proof.

\subsection{Proof of Theorem 2}

Theorem 2 is a direct consequence of our previous results together with [9, Theorem 6$]$. In fact, from Proposition 6 we obtain the equivalence $(i) \Leftrightarrow(i i)$. Theorem 7 implies that $(i)$ is equivalent to $(i i i)$ and that $(i i)$ is equivalent to (iv). Theorem 10 and Corollary 11 give the equivalences $($ ii $) \Leftrightarrow($ viii $)$ and $(i) \Leftrightarrow(v i i)$, respectively. Finally, with $[9$, Theorem 6$]$, we have the equivalences $(i) \Leftrightarrow(v)$ and $(i i) \Leftrightarrow(v i)$. The regularity of $V(\cdot)$ and the conditions on $V^{\prime}(\cdot, \cdot)$ in (vi) actually follow from Lemma 8 and Corollary 9 in [9].

\section{An application of Theorem 2: robustness with respect to perturbations}

In this section we are interested in understanding the effects of small perturbations of the dynamics on the stability of the system. The idea is to exploit the existence of a common Lyapunov-Krasovskii functional. We start by a simple and classical result whose proof is provided for completeness.

Lemma 12 Let $Z$ be a normed space with norm $\|\cdot\|_{Z}$ and $V: Z \rightarrow \mathbb{R}$ be the square of a norm such that $V(x) \leq$ $C\|x\|_{Z}^{2}$ for some $C>0$ and for every $x \in Z$. Then $V \overline{i s}$ locally Lipschitz continuous and satisfies

$$
|V(x)-V(y)| \leq C\left(\|x\|_{Z}+\|y\|_{Z}\right)\|x-y\|_{Z}
$$

Proof. Since $\sqrt{V}$ is a norm, we can apply the triangular inequality so that

$$
|\sqrt{V(x)}-\sqrt{V(y)}| \leq|\sqrt{V(x-y)}| \leq \sqrt{C}\|x-y\|_{Z} .
$$

Thus

$$
\begin{aligned}
|V(x)-V(y)| & =(\sqrt{V(x)}+\sqrt{V(y)})|\sqrt{V(x)}-\sqrt{V(y)}| \\
& \leq C\left(\|x\|_{Z}+\|y\|_{Z}\right)\|x-y\|_{Z} .
\end{aligned}
$$

Let $Q, P$ be a bounded subsets of $\mathcal{L}\left(C\left([-r, 0], \mathbb{R}^{n}\right), \mathbb{R}^{n}\right)$. Here $P$ has to be regarded as a set of bounded perturbations of the operators in $Q$. We are going to consider the case in which the system

$$
\Sigma: \dot{x}(t)=\Gamma(t) x_{t} \quad \Gamma(t) \in Q
$$

is uniformly exponentially stable and to investigate the stability of the perturbed system

$$
\Sigma_{p}: \dot{x}(t)=(\Gamma(t)+\Lambda(t)) x_{t} \quad \Gamma(t) \in Q, \Lambda(t) \in P .
$$

Let $c=\sup _{\Lambda \in P}\|\Lambda\|_{\mathcal{L}\left(C\left([-r, 0], \mathbb{R}^{n}\right), \mathbb{R}^{n}\right)}$ and let $m$ be as in (2). We have the following result.

Lemma 13 For every $t>0$ we have

$$
\left\|T_{\Gamma(\cdot)}(t)-T_{\Gamma(\cdot)+\Lambda(\cdot)}(t)\right\|_{\mathcal{L}\left(C\left([-r, 0], \mathbb{R}^{n}\right)\right)} \leq C(t),
$$

where $C(t)=c \frac{e^{(2 m+c) t}-e^{m t}}{m+c}$.

Proof. Let $\psi \in C\left([-r, 0], \mathbb{R}^{n}\right)$ and let $y(\cdot)=x^{\Gamma}(\cdot)-$ $x^{\Gamma+\Lambda}(\cdot)$, where $x^{\Gamma}$ and $x^{\Gamma+\Lambda}$ are, respectively, the solutions of $\Sigma$ and $\Sigma_{p}$ with initial condition $\psi$. For $t \geq 0$ and $\theta \in[-r, 0]$ we have

$$
\begin{aligned}
& |y(t+\theta)|=\int_{0}^{\max \{0, t+\theta\}}\left|\Gamma(s) x_{s}^{\Gamma}-(\Gamma(s)+\Lambda(s)) x_{s}^{\Gamma+\Lambda}\right| d s \\
& \leq \int_{0}^{\max \{0, t+\theta\}}\left|\Gamma(s) y_{s}\right| d s+\int_{0}^{\max \{0, t+\theta\}}\left|\Lambda(s) x_{s}^{\Gamma+\Lambda}\right| d s \\
& \leq m \int_{0}^{\max \{0, t+\theta\}}\left\|y_{s}\right\|_{C} d s+c \int_{0}^{\max \{0, t+\theta\}}\left\|x_{s}^{\Gamma+\Lambda}\right\|_{C} d s \\
& \leq m \int_{0}^{\max \{0, t+\theta\}}\left\|y_{s}\right\|_{C} d s+c \int_{0}^{\max \{0, t+\theta\}}\|\psi\|_{C} e^{(m+c) s} d s \\
& \leq m \int_{0}^{\max \{0, t+\theta\}}\left\|y_{s}\right\|_{C} d s+c \frac{e^{(m+c) t}-1}{m+c}\|\psi\|_{C} .
\end{aligned}
$$

This leads to

$\left\|y_{t}\right\|_{C} \leq m \int_{0}^{t}\left\|y_{s}\right\|_{C} d s+c \frac{e^{(m+c) t}-1}{m+c}\|\psi\|_{C}$,

from which we obtain, thanks to Gronwall's Lemma

$\left\|y_{t}\right\|_{C} \leq c \frac{e^{(2 m+c) t}-e^{m t}}{m+c}\|\psi\|_{C}$

Thus, we get the thesis.

Corollary 14 Let $V: C\left([-r, 0], \mathbb{R}^{n}\right) \rightarrow \mathbb{R}$ be the square of a norm such that $V(\psi) \leq \bar{c}\|\psi\|_{C}^{2}$ for some $\bar{c}>0$ and for every $\psi \in C\left([-r, 0], \mathbb{R}^{n}\right)$. Then

$$
\bar{D}_{L+\Lambda} V(\psi) \leq \bar{D}_{L} V(\psi)+2 \bar{c} c\|\psi\|_{C}^{2},
$$

$\forall L \in Q, \forall \Lambda \in P, \forall \psi \in C\left([-r, 0], \mathbb{R}^{n}\right)$ 
Proof. Thanks to the previous lemma we have

$$
\begin{aligned}
& \left|V\left(T_{L+\Lambda}(t) \psi\right)-V\left(T_{L}(t) \psi\right)\right| \\
& \leq \bar{c}\left(\left\|T_{L+\Lambda}(t) \psi\right\|_{C}+\left\|T_{L}(t) \psi\right\|_{C}\right)\left\|T_{L+\Lambda}(t) \psi-T_{L}(t) \psi\right\|_{C} \\
& \leq \bar{c} e^{m t}\left(1+e^{c t}\right)\|\psi\|_{C}\left\|T_{L+\Lambda}(t) \psi-T_{L}(t) \psi\right\|_{C} \\
& \leq 2 \bar{c} C(t) e^{(m+c) t}\|\psi\|_{C}^{2}
\end{aligned}
$$

Since $C(0)=0$ and $C^{\prime}(0)=c$ we have

$$
\bar{D}_{L+\Lambda} V(\psi) \leq 2 \bar{c} c\|\psi\|_{C}^{2}+\bar{D}_{L} V(\psi) .
$$

\subsection{Example}

Consider the scalar time-varying delay system

$$
\dot{x}(t)=-x(t-\tau(t))
$$

where $\tau(\cdot)$ is piecewise constant and takes values in $[0, r]$. System (53) is of the type (3) with $Q=\left\{L_{\tau} \mid \tau \in\right.$ $[0, r]\}$ and $L_{\tau} \varphi=-\varphi(-\tau)$. It is known that (53) is uniformly exponentially stable in $C([-r, 0], \mathbb{R})$ if and only if $r<3 / 2$ (see, e.g., $[15,17]$ ).

Fix $r<3 / 2$ and consider the perturbed system

$$
\dot{x}(t)=-x(t-\tau(t))+\int_{-\bar{r}}^{0} a(s) x(t+s) d s,
$$

where $a \in L^{1}([-\bar{r}, 0], \mathbb{R})$ and $\bar{r} \geq r$ (notice that $\bar{r}$ may be larger than $3 / 2$ ). We claim that, as a straightforward consequence of our converse Lyapunov-Krasovskii theorem and of Corollary 14, system (54) is uniformly exponentially stable in $C([-\bar{r}, 0], \mathbb{R})$ if $\|a\|_{L^{1}}$ is small enough. Let $\Lambda: C([-\bar{r}, 0], \mathbb{R}) \rightarrow \mathbb{R}$ be defined by

$$
\Lambda \psi=\int_{-\bar{r}}^{0} a(s) \psi(s) d s
$$

and notice that $|\Lambda \psi| \leq\|a\|_{L^{1}}\|\psi\|_{C}$. Hence,

$$
\begin{aligned}
\bar{D}_{L+\Lambda} V(\psi) & \leq \bar{D}_{L} V(\psi)+2 \bar{c}\|a\|_{L^{1}}\|\psi\|_{C}^{2} \\
& \leq\left(-1+2 \bar{c}\|a\|_{L^{1}}\right)\|\psi\|_{C}^{2}
\end{aligned}
$$

where $V$ is as in Theorem 2, Item (v). If $\|a\|_{L^{1}}<1 /(2 \bar{c})$ then $(54)$ is uniformly exponentially stable in $C([-\bar{r}, 0], \mathbb{R})$.

\section{Conclusion}

In this work we give a collection of converse LyapunovKrasovskii theorems for uncertain retarded functional differential equations. These results are summarized by Theorem 2. They are obtained using the switched

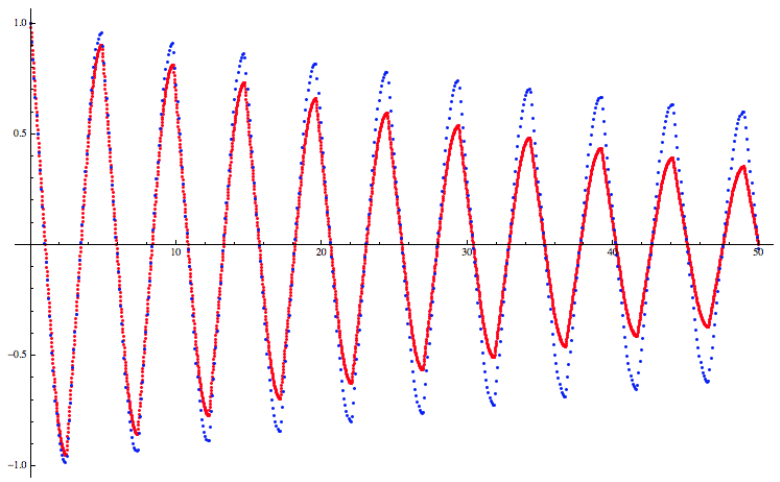

Fig. 2. A solution of (53) (solid line) and a perturbation of it, solution of (54) (dotted line)

system representation approach. One of the novelties of our results is that they use Lyapunov-Krasovskii functionals which may not have a strictly positive normdependent lower bound, in contrast with what is known in the literature.

Concerning the differences between conditions $(v)$ (respectively $(v i))$ and (vii) (respectively (viii)) appearing in the statement of Theorem 2, we already noticed that a Lyapunov function $V(\cdot)$ satisfying condition (vii) (respectively (viii)) does not necessarily satisfy the stronger condition $(v)$ (respectively $(v i)$ ). Hence, condition (vii) (respectively (viii)) is better suited for proving the global uniform exponential stability of a linear uncertain retarded differential equations, while condition $(v)$ (respectively $(v i)$ ) provides more information on a linear uncertain retarded differential equations that is known to be globally uniformly exponentially stable, by tightening the properties satisfied by $V(\cdot)$.

\section{A Appendix: a technical lemma}

Lemma 15 Let $Q \subset \mathcal{L}\left(C\left([-r, 0], \mathbb{R}^{n}\right), \mathbb{R}^{n}\right)$ be bounded. Take $T>0$ and let $x(\cdot)$ and $x_{k}(\cdot)$ be the unique solutions of systems (3) and (33), respectively, where $\Gamma_{k}(\cdot)$ is as in the proof of Theorem 7 and having the same initial condition $x_{0}=x_{k, 0} \in C\left([-r, 0], \mathbb{R}^{n}\right)$. Then

$$
\lim _{k \rightarrow+\infty}\left\|x_{k, T}-x_{T}\right\|_{C}=0
$$

Proof. Let $y_{k}(\cdot)=x_{k}(\cdot)-x(\cdot)$ and $y_{k, t}=x_{k, t}-x_{t}$. By integrating systems (3) and (33) we have that, for every $\theta \in[-r, 0]$ such that $T+\theta>0$,

$$
y_{k}(T+\theta)=\int_{0}^{T+\theta}\left(\Gamma_{k}(s) x_{k, s}-\Gamma(s) x_{s}\right) d s
$$


Adding and subtracting $\int_{0}^{T+\theta} \Gamma_{k}(s) x_{s} d s$ to the previous equation, and using equation (2), we obtain

$$
\left|y_{k}(T+\theta)\right| \leq m \int_{0}^{T+\theta}\left\|y_{k, s}\right\|_{C} d s+\left|f_{k}(T+\theta)\right|,
$$

with $f_{k}(t)=\int_{0}^{t}\left(\Gamma_{k}(s)-\Gamma(s)\right) x_{s} d s$. This leads to

$$
\left\|y_{k, T}\right\|_{C} \leq m \int_{0}^{T}\left\|y_{k, s}\right\|_{C} d s+\left\|f_{k}\right\|_{C[0, T]}
$$

from which we obtain, thanks to Gronwall's Lemma, that

$$
\left\|y_{k, T}\right\|_{C} \leq e^{m T}\left\|f_{k}\right\|_{C[0, T]}
$$

We are going to show that $f_{k}$ converges uniformly to zero on the interval $[0, T]$. Observe that

$$
\begin{aligned}
f_{k}(t)= & \sum_{j=1}^{k} \int_{I_{j} \cap[0, t]}\left(\Gamma_{k}(s)-\Gamma(s)\right)\left(x_{s}-x_{j \frac{T}{k}}\right) d s \\
& -\sum_{j=1}^{k} \underbrace{\int_{I_{j} \cap[0, t]}\left(\Gamma_{k}(s)-\Gamma(s)\right) x_{j \frac{T}{k}} d s}_{K_{j}} .
\end{aligned}
$$

We distinguish three different cases:

- if $j<\left[\frac{k t}{T}\right]$, then by definition of $\Gamma_{k}(\cdot)$, we have

$$
\begin{aligned}
K_{j} & =\int_{I_{j}} \Gamma_{k}(s) x_{j \frac{T}{k}} d s-\int_{I_{j}} \Gamma(s) x_{j \frac{T}{k}} d s \\
& =\sum_{i=1}^{n+1} \int_{I_{i j}} L_{i j} x_{j \frac{T}{k}} d s-\frac{T}{k} \sum_{i=1}^{n+1} \alpha_{i j} L_{i j} x_{j \frac{T}{k}} \\
& =\sum_{i=1}^{n+1} \alpha_{i j} \frac{T}{k} L_{i j} x_{j \frac{T}{k}}-\frac{T}{k} \sum_{i=1}^{n+1} \alpha_{i j} L_{i j} x_{j \frac{T}{k}} \\
& =0
\end{aligned}
$$

- if $j=\left[\frac{k t}{T}\right]$, then

$$
\begin{aligned}
K_{j} & =\int_{\left[(j-1) \frac{T}{k}, t\right]}\left(\Gamma_{k}(s)-\Gamma(s)\right) x_{j \frac{T}{k}} d s \\
& \leq \int_{\left[(j-1) \frac{T}{k}, t\right]}\left|\left(\Gamma_{k}(s)-\Gamma(s)\right) x_{j \frac{T}{k}}\right| d s \\
& \leq 2 m \int_{I_{j}}\left\|x_{j \frac{T}{k}}\right\|_{C} d s \\
& =2 m \frac{T}{k}\left\|x_{j \frac{T}{k}}\right\|_{C}
\end{aligned}
$$

- if $j>\left[\frac{k t}{T}\right]$, then $I_{j} \cap[0, t]=\emptyset$, and by consequence $K_{j}=0$.
Since $x(\cdot)$ is uniformly continuous on $[0, T]$, we deduce that for every $\epsilon>0$ there exists $k_{0}$ such that if $k \geq k_{0}$ then $\sum_{j=1}^{k}\left|K_{j}\right|<\epsilon$. Moreover, there exists $k_{1}$ such that if $k \geq k_{1}$ then $\left\|x_{s}-x_{j \frac{T}{k}}\right\|_{C}<\epsilon$ for every $s \in I_{j}$ and every $j=1, \ldots, k$. As a consequence, if $k \geq \max \left\{k_{0}, k_{1}\right\}$ we have

$$
\left|f_{k}(t)\right| \leq(2 m T+1) \epsilon
$$

Note that this is true for every $t \in[0, T]$. We conclude that

$$
\left\|f_{k}\right\|_{C[0, T]} \leq(2 m T+1) \epsilon .
$$

Equation (A.3), together with (A.2), concludes the proof of (A.1).

\section{References}

[1] L. Bao, S. Fei, and L. Yu. Exponential stability of linear distributed parameter switched systems with time-delay. $J$. Syst. Sci. Complex., 27(2):263-275, 2014.

[2] R. Curtain and H. Zwart. An introduction to infinitedimensional linear systems theory, volume 21. SpringerVerlag, 1995.

[3] E. Fridman. Stability of systems with uncertain delays: a new "complete" Lyapunov-Krasovskii functional. IEEE Transactions on Automatic Control, 51(5):885-890, 2006.

[4] E. Fridman and S. I. Niculescu. On complete LyapunovKrasovskii functional techniques for uncertain systems with fast-varying delays. Int. J. Robust Nonlinear Control, 18(3):364-374, 2008.

[5] E. Fridman, U. Shaked, and K. Liu. New conditions for delay-derivative-dependent stability. Automatica J. IFAC, 45(11):2723-2727, 2009.

[6] K. Gu, V. L. Kharitonov, and J. Chen. Stability of time-delay systems. Birkhäuser, 2003.

[7] J. W. Hagood and B. S. Thomson. Recovering a function from a Dini derivative. Amer. Math. Monthly, 113:34-46, 2006.

[8] J. Hale and S. V. Lunel. Introduction to functional differential equations, volume 99. Springer-Verlag, 1993.

[9] F. Hante and M. Sigalotti. Converse Lyapunov theorems for switched systems in Banach and Hilbert spaces. SIAM J. Control Optim., 49(2):752-770, 2011.

[10] L. Hetel, J. Daafouz, and C. Iung. Equivalence between the Lyapunov-Krasovskii functional approach for discrete delay systems and the stability conditions for switched systems. Nonlinear Analysis: Hybrid Systems, 2(3):697-705, 2008.

[11] W. Huang. Generalization of Liapunov's theorem in a linear delay system. Journal of Mathematical Analysis and Applications, 142:83-94, 1989.

[12] I. Karafyllis. Lyapunov theorems for systems described by retarded functional differential equations. Nonlinear Analysis: Theory, Methods \& Applications, 64:590-617, 2006.

[13] I. Karafyllis, P. Pepe, and Z.-P. Jiang. Global output stability for systems described by retarded functional differential equations: Lyapunov characterizations. Eur. J. Control, 14(6):516-536, 2008. 
[14] V. L. Kharitonov and A. P. Zhabko. Lyapunov-Krasovskii approach to the robust stability analysis of time-delay systems. Automatica, 39(1):15-20, 2003.

[15] J. C. Lillo. Oscillatory Solutions of the Equation $y^{\prime}(x)=$ $m(x) y(x-n(x))$. Journal Of Differential Equations, 6:1-35, 1969.

[16] F. Mazenc, S. I. Niculescu, and M. Krstic. LyapunovKrasovskii functionals and application to input delay compensation for linear time-invariant systems. Automatica J. IFAC, 48(7):1317-1323, 2012.

[17] A. D. Myshkis. On solutions of linear homogeneous differential equations of the first order of stable type with a retarded argument (in Russian). Mat. Sb. (N.S.), 28(70)(3):641-658, 1951.

[18] S. I. Niculescu. Delay effects on stability: A robust control approach, volume 269. Berlin: Springer, 2001.

[19] P. Pepe and I. Karafyllis. Converse Lyapunov-Krasovskii theorems for systems described by neutral functional differential equations in Hale's form. International Journal of Control, 86(2):232-243, 2013.

[20] J. P. Richard. Time-delay systems: An overview of some recent advances and open problems. Automatica, 39:16671694, 2003.

[21] A. Sasane. Stability of switching infinite-dimensional systems. Automatica J. IFAC, 41(1):75-78, 2005.

[22] R. Triggiani. A sharp result on the exponential operatornorm decay of a family of strongly continuous semigroups. Semigroup Forum, 49(2):387-395, 1994. 